\title{
LA GLOBALIZZAZIONE DELLA DISEGUAGLIANZA. ASPETTI TEORICI, EMPIRICI E DI POLICY
}

\author{
Nota del s.c. RENATA TARGETTI LENTI (*)
}

(Adunanza del 10 aprile 2014)

\begin{abstract}
SunTO. - A partire dall'inizio degli anni '90 la diseguaglianza è tornata ad essere una delle tematiche centrali del dibattito economico sotto diversi profili: teorico, applicato e di policy. E' cresciuta inoltre l'attenzione non solo per la diseguaglianza all'interno dei paesi, ma anche di quella globale, e cioè di quella tra i paesi e tra i cittadini del mondo considerati come appartenenti ad una unica comunità. Gli effetti della globalizzazione sulla diseguaglianza sono tuttora molto controversi. Secondo alcuni autori l'intesificarsi del processo di integrazione internazionale ha prodotto non solo instabilità e crisi ricorrenti, ma anche una crescente diseguaglianza all'interno e tra paesi. Secondo altri, invece, diseguaglianza e povertà si sono ridotte proprio grazie alla globalizzazione Il presente lavoro si propone di analizzare il tema della diseguaglianza globale principalmente dal punto di vista empirico. In via preliminare, tuttavia, saranno discusse alcune questioni relative alla definizione del fenomeno con riferimento sia agli aspetti teorici sia a quelli normativi. L'analisi empirica sarà condotta distinguendo il peso sulla diseguaglianza globale della componente tra paesi (between) rispetto a quella interna ai paesi (within). Non ci si limiterà ad analizzare le variazioni degli indici sintetici, ma si evidenzieranno le differenze nella distribuzione dei redditi dei singoli paesi. Questo tipo di analisi, innovativa rispetto a quanto viene generalmente proposto, permetterà di osservare come proprio le differenze distributive tra paesi industrializzati e paesi in via di sviluppo siano in grado di dar conto di fenomeni importanti a livello internazionale come, ad esempio, i flussi migratori.
\end{abstract}

$$
* * *
$$

ABSTRACT. - Since the beginning of the 90's inequality, once again, become one of the central issues of the economic debate from different perspectives: theoretical, applied

(*) Università degli Studi di Pavia, Italia. E-mail: targetti@unipv.it 
and of policy. Not only increased the attention toward the inequality within countries, but also toward the global one, that is the inequality between countries and between citizens of the world as they belong to a single community. The effects of globalization on inequality are still very controversial. According to some authors international integration has produced not only instability and recurring crises, but also a growing inequality within and between countries. For other authors, instead, inequality and poverty decreased with the globalization. This paper will analyze the issue of global inequality mainly from an empirical standpoint. First of all, however, it will be discussed some issues related to the definition of the phenomenon with reference to the theoretical as well to the normative aspects. The empirical analysis will be undertaken by distinguishing the weight of the inequality between countries from that within countries on global inequality. Changes of synthetic indexes will be calculated, but also the differences in income's distribution in each country will be analyzed. This kind of analysis, innovative with respect to the traditional ones, will allow to observe how the differences in the income's distribution of industrialized and of developing countries can justify phenomena of the global economy such as, for example, migratory flows.

\section{INTRODUZIONE}

A partire dall'inizio degli anni '90 la "diseguaglianza", in particolare quella nella distribuzione dei redditi, è tornata ad essere una delle tematiche centrali del dibattito economico sotto diversi profili: teorico, applicato e di policy (Atkinson, 1997). Il tema ha acquistato inoltre, negli ultimi anni, nuove dimensioni all'interno di ogni paese in relazione alle trasformazioni dei rapporti sociali e personali ed a livello internazionale con l'intensificarsi dei processi di globalizzazione. E' cresciuta l'attenzione non solo per la diseguaglianza all'interno dei paesi, ma anche per quella globale, e cioè per quella tra paesi e tra cittadini del mondo considerati come appartenenti ad una unica comunità. Amartya Sen $(2002$, p. 5) ha sottolineato come la sfida principale abbia oggi a che fare "in un modo o nell'altro, con la disuguaglianza, sia tra le nazioni sia nelle nazioni....Una questione cruciale è la divisione, tra paesi ricchi e paesi poveri o tra differenti gruppi in un paese, dei guadagni potenziali generati dalla globalizzazione". Se alcuni paesi hanno progressivamente ridotto la distanza fra il valore del reddito medio rispetto e quello medio mondiale, altri hanno invece ampliato tale divario.

Gli effetti della globalizzazione sulla diseguaglianza sono tuttora molto controversi. Secondo alcuni autori l'intesificarsi del processo di integrazione internazionale ha prodotto non solo instabilità e crisi ricorrenti, ma anche una crescente diseguaglianza all'interno e tra i paesi 
(Stiglitz, 2002). Secondo altri diseguaglianza e povertà si sono invece ridotte proprio grazie alla globalizzazione (Anand, Segal, 2008). Il Segretario Generale delle Nazioni Unite Kofi Annan ha affermato che "the main losers in today's very unequal world are not those who are too much exposed to globalization. They are those who have been left out" (IMF, 2008, p. 7). Secondo, tra gli altri, Dollar e Kray (2004) proprio l'intensificarsi degli scambi internazionali avrebbe contribuito allo sviluppo economico di molti paesi ed in particolare dei cosiddetti globalisers. I fattori che hanno contribuito alla crescita della diseguaglianza globale sono numerosi e di varia natura. Alcuni dei fattori all'origine del mutamento della diseguaglianza sono specifici ed endogeni ai diversi contesti nazionali, altri sono invece da considerarsi esogeni e cioè dipendenti dalla globalizzazione.

Il presente lavoro si propone di analizzare il tema della diseguaglianza globale principalmente dal punto di vista empirico. In via preliminare, tuttavia, saranno discusse alcune questioni relative alla definizione del fenomeno con riferimento sia agli aspetti teorici sia a quelli normativi. L'analisi empirica sarà condotta distinguendo il peso sulla diseguaglianza globale della componente tra paesi (between) rispetto a quella interna ai paesi (within). Non ci si limiterà ad analizzare le variazioni degli indici sintetici, ma si evidenzieranno le differenze nella distribuzione dei redditi dei singoli paesi. Questo tipo di analisi, innovativa rispetto a quanto viene generalmente proposto, permetterà di osservare come proprio le differenze distributive tra paesi industrializzati e paesi in via di sviluppo siano in grado di dar conto di fenomeni importanti a livello internazionale come, ad esempio, i flussi migratori (Milanovic, 2011b, 2012).

\section{DiseguaglianZa E TEORIE DELla GIUSTIZIA DistributiVA}

Il dibattito sulla diseguaglianza e sulla equità, intesa come parametro di riferimento per le scelte sociali, si è sviluppato lungo direzioni alternative. In economia politica l'analisi è stata, per così dire, confinata ad una sola branca, l'economia del benessere. La principale motivazione teorica che ha spinto a studiare la diseguaglianza economica riguarda la natura della relazione tra efficienza ed equità, all'interno della determinazione di una funzione del benessere collettivo. Com'è noto, nell'ambito dell'impostazione neoclassica ed in particolare di quella 
paretiana, l'efficienza è considerata un obiettivo, da raggiungersi al fine di ottimizzare il processo produttivo, prioritario rispetto all'equità. Da qui un rapporto di subordinazione della seconda rispetto alla prima. L'equità, e dunque l'eguaglianza, può essere considerata al massimo come un vincolo da rispettare in un mercato perfettamente concorrenziale. Secondo autorevoli autori come Robbins (1932) lo studio dell'economia politica dovrebbe esaurirsi nello studio dell'efficienza, o meglio delle scelte razionali da attuarsi in presenza di risorse scarse da utilizzarsi per fini alternativi. I progressi dell'economia del benessere hanno portato a riconsiderare la relazione fra efficienza ed equità. In particolare non sembra più accettabile l'ipotesi che si possano mantenere separati i due concetti, specialmente se si tiene conto dell'esistenza di asimmetrie informative e di imperfezioni di mercato (Kanbur, Lustig, 1999). Ne consegue che l'obiettivo di un'equa ripartizione delle risorse, ed in particolare del reddito, diventano parti integranti del funzionamento del sistema economico e non solo un vincolo da rispettare una volta conseguita l'efficienza.

Il primo dei due teoremi "fondamentali" dell'economia del benessere, formulati da Arrow e Debreu (1954), prova che un equilibrio competitivo non è dominato in senso paretiano da nessuna altra allocazione socialmente fattibile, ovvero corrisponde alla frontiera della massima efficienza produttiva. Il secondo teorema, invece, dimostra che, data una qualsiasi allocazione delle risorse ottimale in senso paretiano, è sempre possibile, sotto certe condizioni, individuare dei meccanismi per redistribuire le risorse tra gli individui così che l'allocazione di equilibrio walrasiano relativa a quella distribuzione (più egualitaria) coincida con l'allocazione data. I due sottoproblemi che immeditamente sorgono riguardano: i) la scelta della combinazione di risorse che corrisponda ad una allocazione non necessariamente egualitaria, ma più equa con riferimento ad una determinata funzione del benessere collettivo; ii) la combinazione di tasse o trasferimenti che garantiscano che sia possibile raggiungere l'obiettivo socialmente desiderabile partendo da una determinata allocazione iniziale.

Con riferimento al primo problema ci si trova di fronte ad una molteplicità di teorie della giustizia distributiva a cui è possibile far corrispondere funzioni del benessere collettivo alternative. La pubblicazione nel 1971 da parte di John Rawls di A Theory of Justice, ha avviato un dibattito particolarmente ricco, a cui hanno partecipato non solo filosofi ma anche numerosi economisti, sui fondamenti etici delle politiche 
redistributive. Sono state proposte teorie alternative della giustizia distributiva in relazione al concetto di giusto e di bene. Si è messo in luce come il tentativo di favorire l'eguaglianza in uno spazio valutativo possa creare un maggior grado di diseguaglianza in altri spazi. D'altra parte l'eguaglianza è perseguita in determinate sfere in quanto viene considerata necessaria, o comunque strumentale, alla realizzazione dell'eguaglianza in qualche altra sfera più importante, nella quale è ritenuta essenziale. L'esempio più evidente è quello di rendere effettivi certi diritti o libertà fondamentali formalmente uguali, ma il cui esercizio sostanziale dipende da una serie di condizioni materiali delle quali i soggetti dispongono in misura ineguale.

L'atteggiamento verso la diseguaglianza che la società è disposta a tollerare o a ritenere giustificabile svolge un ruolo molto importante nello spiegare il livello di diseguaglianza che si instaura in realtà nei diversi sistemi economici. Cambiamenti nella forma della funzione del benessere sociale si manifestano, ad esempio, nell'alternanza degli schieramenti politici al governo: un governo di impostazione liberale sarà maggiormente propenso a tollerare un elevato livello di diseguaglianza rispetto ad uno socialdemocratico. In paesi diversi prevalgono norme sociali anche molto differenti: tipico è, non a caso, il confronto tra Stati Uniti da un lato e paesi dell'Europa continentale dall'altro. La società americana è percepita, dagli stessi americani, come più diseguale, ma anche come più efficiente e meritocratica. La Cina fornisce un esempio estremo dell'importanza delle norme sociali: nel corso degli ultimi due decenni l'atteggiamento nei confronti dell'economia di mercato, a tutti i livelli della società, è radicalmente mutato, e contemporaneamente la diseguaglianza interna è aumentata in modo significativo.

Dall'impostazione normativa appena delineata discende l'esigenza di attuare politiche appropriate per ridurre la diseguaglianza e la povertà all'interno dei diversi paesi. Non vi è dubbio che lo sviluppo della democrazia sia stato accompagnato dall'affermarsi di movimenti politici che hanno posto al centro dei propri programmi l'eguaglianza. In molti paesi il consolidamento delle istituzioni democratiche ed il rispetto delle libertà fondamentali è stato accompagnato dall'introduzione di meccanismi redistributivi e dalla formazione di sistemi di "welfare state che hanno consentito di realizzare, in termini diversi da paese a paese e con esiti non sempre univoci, alcune parti dei progetti ugualitari". Tuttavia il "tentativo di ottenere rapidi e decisivi risultati sul versante dell'uguaglianza ha portato in alcuni casi ad abbandonare il sen- 
tiero democratico, o a scartarlo prima ancora di averlo imboccato" (Somaini, 2002, p. XI). All'interno dei diversi paesi, ed in particolare in quelli europei, le istanze egualitarie si sono tradotte nella protezione di alcuni gruppi di lavoratori (gli insider), che godono delle protezioni dei sistemi di welfare e della rappresentanza sindacale, in contrapposizione, se non addirittura in conflitto, con i gruppi al di fuori di queste istituzioni (gli outsider).

\section{PerchÈ OCCUPARSI DI DiSEguaglianZA GLOBALE}

Secondo autori come Pogge (1994, 2008), Reddy $(1005,2011)$ o Singer (2004) sono motivazioni etiche quelle che inducono a considerare come "ingiusta" non solo la diseguaglianza interna ai diversi paesi, ma anche quella globale, e dunque spingono ad occuparsi della distribuzione del reddito a livello internazionale e cioè tra gli individui, considerati come cittadini del mondo. Negli anni ' 90 le ricerche e le applicazioni di una qualche prospettiva etica $\mathrm{o}$, più semplicemente, dal punto di vista etico alla politica e alle politiche si sono rivolte al tentativo di una estensione della portata "dei principi di giustizia dal contesto interno delle comunità all'arena della comunità internazionale" (Veca, 2001, p. 2). Per Veca, tra gli altri, occorre immaginare "un'etica per la politica, le istituzioni e le pratiche sociali" che possano valere "per chiunque... indipendentemente da confini dati o dalla sorte contingente per cui ci è accaduto di nascere qua o là in un mondo sempre più piccolo e interdipendente". La questione può essere così impostata: "come rispondere all'iniquità globale dal punto di vista della giustizia e dell'ingiustizia, piuttosto che del solo punto di vista umanitario?" (Veca, 2001, p. 5).

Il problema che si pone non è solo quello dell'estensione di alcuni valori come quello della pace o del rispetto dei diritti umani al contesto internazionale. Se così fosse potremmo trovare risposte in Rawls nel suo The Law of peoples (1999), o in Nagel (2005) quando sostiene la desiderabilità di istituzioni internazionali o sopranazionali. Il problema, ancora poco esplorato, riguarda l'estensione, al di fuori dei confini nazionali, dei principi di giustizia che dovrebbero regolare il conflitto distributivo. La maggior parte delle teorie della giustizia, dall'utilitarismo al contrattualismo al libertarismo assumono come contesto rilevante quello nazionale.... Esse "possono essere riconsiderate, in modo piano e naturale, come teorie centrate su risposte diverse alla stessa domanda di giustifi- 
cazione etica delle istituzioni e delle pratiche sociali...." (Veca, 2001, p. 1). I due principi di giustizia che sono alla base della teoria della giustizia di Rawls (di natura contrattualistica) difficilmente potrebbero valere ed essere estesi dal contesto nazionale a quello internazionale. Secondo autori come Bhagwati (2004) proprio la mancanza di un governo globale e quindi anche di una "global polity" (Milanovic, 2006, p. 148) rende il concetto di diseguaglianza a livello globale privo di significato dal punto di vista normativo. Nel contesto internazionale manca, infatti, un'Istituzione in grado di assicurare il rispetto di quel contratto tra cittadini, che è il fondamento della teoria della giustizia di Rawls.

Secondo altri autori, invece, numerose motivazioni giustificano lo studio della diseguaglianza globale Per Sen queste motivazioni sono da ricercarsi, innanzitutto, nel nesso tra sviluppo e libertà, e cioè nel fatto che la crescita del prodotto interno lordo o dei redditi individuali possono essere molto importanti come mezzi per espandere le libertà delle persone che fanno parte di una società (Sen, 2001). Anche per Veca l'idea di sviluppo come libertà può costituire "un criterio normativo e valutativo universalistico e non dipendente da contesti, politicamente neutrale, che ci orienti nel giudizio politico riflessivo quando cerchiamo di guadagnare una prospettiva plausibile su questioni di giustizia globale" (Veca, 2001, p. 9).

L'impostazione che sembra essere più feconda, a questo scopo, è quella che Sen ha recentemente discusso nel contributo Contro l'ingiustzia. In esso ripropone molti dei temi oggetto del precedente, ampio e complesso, volume L'idea di giustizia (Sen, 2010). La dimensione etica e l'attenzione per la rilevanza sociale dei problemi economici pervadono tutta l'opera di Sen e caratterizzano anche questi ultimi due lavori (Targetti Lenti, 2010). Naturalmente è impossibile dare conto in sintesi della ricchezza dell'impostazione di Sen. Mi limiterò qui a discutere alcuni punti che ritengo essenziali per giustificare, dal punto di vista non solo normativo ma anche positivo, l'estensione al contesto internazionale dei principi di giustizia validi per quello nazionale. Come lo stesso Sen sottolinea, l'intento non può essere quello di formulare una teoria della giustizia "astratta" concentrata sull'identificazione di un "istituzionalismo trascendentale" riconducibile alla tradizione illuminista, ma invece quello di adottare un approccio comparativo connesso "con le concrete realizzazioni sociali (frutto delle istituzioni reali, dei reali comportamenti e di altri fattori)" (Sen, 2010, p. 21).

La teoria della scelta sociale è considerata l'impostazione più 
adatta per costruire una teoria della giustizia. Questa scelta è giustificata non solo dal fatto di essere Sen un economista, ma perché le questioni di giustizia devono essere analizzate al fine di disegnare politiche atte a ridurre, se non eliminare, le ingiustizie più gravi, sia a livello nazionale che internazionale, migliorando così il tenore di vita delle persone. I "principi di giustizia" non sono "definiti in rapporto alla nozione di istituzioni perfettamente giuste”, ma invece scegliendo di accordare priorità ad un interrogativo quale "come è possibile promuovere la giustizia” (Sen, 2010, p. 25). Sen chiarisce inoltre la sua posizione rispetto a Rawls affermando che la sua impostazione riguarda la possibilità di "giungere ad accordi sulla riduzione dell'ingiustizia razionali e, allo stesso tempo, perseguibili nonostante le diverse idee sulla forma di governo ideale" (Sen, 2010, p. 28). Il progetto seniano è perseguito, innanzitutto, facendo uso dello strumento "dell'imparzialità" nel confronto pubblico. Nello spazio globale diviene fondamentale il riferimento al carattere aperto e non chiuso della virtù dell'imparzialità, sostenuta nella sua Teoria dei sentimenti morali da Adam Smith" (Sen, 2010, p. 83). Lidea di imparzialità aperta sembra essere particolarmente appropriata per una teoria della giustizia globale.

Quali siano i fattori che determinano le ingiustizie, all'origine delle diverse forme di diseguaglianza e dunque anche di quelle di reddito, resta la domanda di fondo alla quale non è stata ancora fornita una risposta soddisfacente. Le ingiustizie sono, secondo Sen, in larga misura eliminabili attraverso adeguate politiche sociali. Il raggiungimento di questo obiettivo richiede l'eliminazione dei numerosi ostacoli (culturali, religiosi, istituzionali) che creano discriminazione tra i diversi individui in relazione alle caratteristiche personali (sesso, razza, presenza di handicap) e ambientali (malattie endemiche, inquinamento). Queste caratteristiche sono da considerarsi in larga misura casuali ovvero determinate "dalla lotteria del destino" (Sen, 1994, p. 97).

Altre importanti ragioni, economiche e socio-demografiche, spingono ad occuparsi della diseguaglianza non solo a livello nazionale, ma anche internazionale (Anand, Segal, 2008, p. 59). Un importante fenomeno come quello delle migrazioni, ad esempio, trova giustificazione nelle enormi differenze di reddito e di livello di vita tra i diversi paesi. Parallelamente al processo di globalizzazione sono aumentati i flussi migratori, non solo da Sud a Nord, ma anche tra paesi industrializzati. Flussi migratori consistenti dall'Europa agli Stati Uniti o verso l'America del Sud hanno accompagnato, ad esempio, la prima grande 
ondata di globalizzazione (Cornia, 2004). Sempre la ricerca di un'occupazione spiega, oggi, il flusso di lavoratori a basso livello di qualificazione dal Messico, dal Sud America o dall'Asia verso gli Stati Uniti nonché dal Nord Africa o dal Medio Oriente verso l'Europa.

Nella teoria del commercio internazionale le migrazioni sono considerate il fattore che dovrebbe servire a livellare le retribuzioni, e dunque il costo del lavoro tra paesi. Il fenomeno migratorio, tuttavia, ha assunto oggi caratteri parzialmente diversi. Sempre più spesso lavoratori ad elevato livello di qualificazione si spostano tra paesi europei. Il fattore determinante, in questo caso, non è solo la ricerca di un'occupazione generica, ma di un'occupazione più remunerativa e di qualificazione più elevata. Nello stesso tempo stanno emergendo reazioni di chiusura da parte dei paesi di destinazione. Un fattore importante nello spiegare l'attrattività di alcuni paesi rispetto ad altri resta il differenziale tra remunerazioni, e dunque tra i redditi che è possibile conseguire in un paese rispetto ad un altro. Le diverse forme di diseguaglianza nella distribuzione dei redditi, oltre che nei livelli occupazionali, tra paesi diversi costituisce uno dei fattori esplicativi del fenomeno migratorio. Per questo motivo, dunque, oltre che per motivazione etiche connesse ad un concetto di teoria della giustizia distributiva, è importante analizzare la diseguaglianza tra paesi, e cioè la "diseguaglianza globale" intesa non solo come divario tra paesi, ma anche tra cittadini appartenenti a paesi diversi.

Motivazioni di natura politica, infine, potrebbero indurre ad analizzare la diseguaglianza globale. Il diverso potere decisionale all'interno delle Organizzazioni Internazionali riflette il diverso potere economico, e dunque la diseguaglianza nella dotazione di risorse. Una elevata diseguaglianza influenza negativamente le condizioni di vita delle persone, riduce la coesione sociale all'interno d'ogni paese e tra paesi. E' in questo contesto che si deve collocare, ad esempio, la discussione sulla riforma delle istituzioni finanziarie internazionali e sul ruolo degli aiuti ai paesi in difficoltà.

\section{I FATTORI ALL'ORIGINE DELLA DISEGUAGLIANZA NELLA DISTRIBUZIONE PERSONALE DEI REDDITI}

Il tentativo, sicuramente ambizioso, di costruire una teoria delle giustizia globale può essere accantonato a favore di uno meno ambizio- 
so, che si limiti ad analizzare i fattori all'origine delle diseguaglianze tra le persone non solo all'interno dei singoli paesi, ma anche a livello internazionale. Se si accetta che l'eguaglianza debba essere considerata una delle variabili che determinano l'equilibrio del sistema economico, sarebbe necessario poterla definire così come accade per il concetto di efficienza. In realtà non è semplice, né forse possibile, fornire una definizione univoca di eguaglianza e/o disuguaglianza, dal momento che questa può differire in relazione alla variabile di riferimento (reddito, ricchezza, tenore di vita, utilità, felicità, opportunità), cosicché l'eguaglianza in termini di una variabile può divergere anche in modo significativo dall'eguaglianza valutata con riferimento ad un'altra (Sen, 1994, p. 16). Nell'ambito dell'economia politica la variabile "focale" è generalmente individuata nel reddito e/o nella ricchezza, in quanto variabili più facilmente quantificabili. Essendo esprimibili in termini monetari, esse sono utilizzabili per confronti nel tempo e nello spazio. La grandezza "reddito disponibile", d'altra parte, resta un buon indicatore, anche se non certamente l'unico, del tenore di vita. La scelta di una variabile quantificabile come il reddito, inoltre, consente di identificare indici che misurino la diseguaglianza globale e che possano esseri presi a riferimento di politiche di riduzione della stessa. Occorre dunque, in via preliminare, individuare $\mathrm{i}$ fattori che possono essere considerati all'origine della "diseguaglianza economica" nel processo di formazione dei redditi individuali.

Per molto tempo la distribuzione personale dei redditi è stata considerata un processo stocastico di cui debbono essere determinate le leggi statistiche che lo governano, avendo come obiettivi primari la formulazione di "leggi" generali per descrivere la "forma" della distribuzione e la misura, sulla base di tali leggi, del relativo grado di disuguaglianza: tale modalità di analisi privilegia quindi più gli aspetti statistici di quelli economici. Pareto, in particolare, aveva individuato una relazione tra redditi individuali e numero di percettori, a partire da un valore del reddito minimo, così significativa da potere essere considerata una vera e propria legge. Questa impostazione statistico-descrittiva ha portato ad una sorta di separazione tra gli sviluppi di una teoria della distribuzione personale del reddito ed il "corpus" principale della teoria economica e, segnatamente, delle teorie della distribuzione funzionale. Nel corso del tempo, tuttavia, la distribuzione personale dei redditi è venuta acquistando importanza crescente anche per l'analisi economica non solo con riferimento ai concetti di equità e di benessere 
ma anche allo studio dei comportamenti individuali e collettivi (concernenti il consumo, l'accumulazione di capitale fisico ed umano), alle caratteristiche dell'organizzazione produttiva e del mercato del lavoro nonché a fini di formulazione delle politiche redistributive.

Non v'è dubbio che i processi attraverso cui i redditi individuali e/o familiari si determinano siano molto complessi. La diseguaglianza accertata nella distribuzione personale è la risultante delle diseguaglianze che si instaurano nel momento della formazione delle diverse componenti del reddito. Queste sono riconducibili, sostanzialmente, all'esistenza di percettori di soli redditi da lavoro in contrapposizione a percettori di redditi provenienti anche o solo dalla proprietà di beni capitali e/o di risorse naturali. All'interno della distribuzione dei redditi percepiti dai diversi gruppi di lavoratori si manifestano diseguaglianze determinate, tra l'altro, dalla posizione nella professione, dall'esercizio di determinate mansioni, dalle capacità individuali, dal livello di istruzione, dalle caratteristiche tecnologiche dei diversi settori produttivi. Il mercato, la famiglia e lo Stato possono cioè essere considerati come i tre momenti nei quali si combinano i diversi fattori all'origine della diseguaglianza.

Dalla distribuzione funzionale del reddito (distinta nelle tre grandi categorie dei salari, dei profitti e delle rendite) si perviene a quella personale (primaria e secondaria) attraverso alcuni passaggi. Il primo è costituito dalla formazione e distribuzione del valore aggiunto ai diversi fattori in rapporto alla produzione del prodotto interno e nell'ambito di una specifica struttura del sistema economico per settori di attività, dimensione di impresa, categorie professionali. Questo primo momento riflette sia le caratteristiche macroeconomiche sia le scelte tecnologiche delle imprese, ovvero le variabili che determinano la ripartizione del reddito tra quote settoriali e funzionali.

Il secondo passaggio riflette il processo di distribuzione primaria del reddito dai fattori alle famiglie. La direzione e la grandezza dei flussi riflette a sua volta la struttura proprietaria dei fattori da parte dei singoli individui, raggruppati in unità familiari di diversa composizione e ampiezza. Alcuni di questi fattori, ad esempio i beni capitali, sono generalmente considerati di proprietà della famiglia. La capacità di ottenere un determinato livello di reddito dipende dalle caratteristiche individuali (abilità personali, innate od acquisite, età), ma anche dalla posizione relativa di ogni soggetto all'interno di una determinata struttura socio-economica. 
Per ciascun individuo, inoltre, il peso di ogni categoria di reddito sul reddito complessivo dipenderà dal livello e dalla composizione delle dotazioni (capitale umano e capitale fisico) che possono essere scambiate sul mercato. I modi attraverso cui le dotazioni di fattori si traducono in redditi dipendono dai prezzi (del lavoro dipendente, del lavoro autonomo e del capitale) quali si determinano in relazione alle condizioni strutturali e congiunturali dei diversi mercati. La diseguaglianza risulterà tanto più elevata quanto più la proprietà delle dotazioni, ed in particolare dei beni capitali, è concentrata; quanto maggiore è la dispersione delle remunerazioni dei fattori ed in particolare del lavoro; quanto più l'esclusione dal mercato e l'emarginazione colpiscono sistematicamente alcune componenti della forza lavoro, specifici settori produttivi, singole aree territoriali.

Il terzo momento è quello in cui viene determinato il valore dei redditi disponibili. Se si tiene conto dell'azione redistributiva del settore pubblico, esercitata attraverso il prelievo delle imposte dirette e degli oneri sociali (effettuato sia sui redditi da lavoro che sul risultato lordo di gestione), l'erogazione di prestazioni sociali (pensioni, indennità di disoccupazione, cassa integrazione guadagni, e così via) ed il pagamento degli interessi sul debito pubblico, si giunge a determinare, partendo da quella primaria, la distribuzione secondaria del reddito. Questo terzo momento, logicamente successivo ma spesso contemporaneo al primo, riflette la struttura dei meccanismi redistributivi che legano il settore delle famiglie a quello della Pubblica Amministrazione. Esso riflette meccanismi distributivi che operano attraverso il sistema tributario (più o meno progressivo) e della sicurezza sociale.

Lo schema di analisi appena introdotto evidenzia come ogni agente economico (individuo, impresa, Stato), grazie alla propria posizione all'interno del sistema economico ed alle interazioni con gli altri agenti, contribuisca a determinare la diseguaglianza che caratterizza la distribuzione dei redditi individuali e familiari sia a livello primario che secondario. E' necessario allora evidenziare le relazioni tra diseguaglianza economica e caratteristiche strutturali del sistema, nonché quelle tra diseguaglianza e politiche redistributive. In sede di analisi empirica, occorre dare risposta ai nuovi e numerosi problemi metodologici che si presentano nel momento in cui si intende "misurare" la diseguaglianza e che sono riconducibili, sostanzialmente, alla scelta delle unità di riferimento e degli indicatori. 


\section{LA DISEGUAGLIANZA GLOBALE NEL BREVE E NEL LUNGO PERIODO}

Contributi importanti all'analisi della diseguaglianza globale sono stati forniti dagli studi sugli indici atti alla sua misurazione e sulle tendenze di questi indici nel breve nel lungo periodo. I risultati e le interpretazioni che se ne possono trarre sono spesso contrastanti, e non solo a causa dei metodi di calcolo e degli indici utilizzati per misurare la diseguaglianza, ma soprattutto per le differenze nei dati di partenza, nelle caratteristiche metodologiche delle indagini, nelle ipotesi necessarie a stimare le diverse distribuzioni nazionali, nel numero di paesi inclusi nel campione considerato ed infine in relazione al periodo considerato. Qualunque sia il modello interpretativo prescelto, tutte le indagini in tema di disuguaglianza nella distribuzione personale dei redditi si basano su specifiche evidenze empiriche, nella maggior parte dei casi tratte da indagini campionarie. La significatività statistica del campione, il metodo di trattazione dei dati, la definizione delle variabili (unità di riferimento, componenti di reddito, periodo temporale di riferimento) condizionano l'interpretazione dei risultati. La definizione stessa di reddito non è priva d'ambiguità.

Seguendo la classificazione proposta da Milanovic (2006a, p. 131), si può fare riferimento a tre concetti di diseguaglianza globale tra loro distinti, ai quali corrispondono tre diverse misure complementari, ciascuna idonea a misurarne un aspetto. Il primo (Concept 1) fa riferimento alla "diseguaglianza tra paesi" (Intercountry inequality) misura i divari nei redditi pro-capite dei diversi paesi prescindendo dalla diversa numerosità della popolazione. Dal momento che la popolazione non entra nel calcolo dell'indice ogni paese ha il medesimo peso nella distribuzione globale del reddito. Questo significa che un aumento nel reddito di un paese "piccolo" ha lo stesso effetto sulla variazione della diseguaglianza globale di un paese più grande e più popoloso.

Il secondo concetto (Concept 2) definito come "diseguaglianza internazionale" (International inequality) misura la diseguaglianza globale come divario tra i redditi pro capite dei diversi paesi tenendo conto della numerosità della popolazione, e dunque ponderando con essa i diversi valori del reddito medio.

Il terzo concetto (Concept 3) di "diseguaglianza globale" (Global inequality), infine, misura la diseguaglianza nella distribuzione dei redditi fra i cittadini (individui o famiglie) considerati come appartenenti 
tutti ad un unico territorio: il mondo. L'indice misura le divergenze tra i redditi individuali e non solo tra il reddito medio delle nazioni. Il calcolo della diseguaglianza viene effettuato sulla base di una distribuzione dei redditi individuali indipendentemente dal paese di appartenenza. L'indice così costruito può essere considerato come il "vero" indicatore della diseguaglianza globale intesa come divario tra redditi di diversi paesi in un mondo composto da individui e non solo da nazioni.

I problemi che si presentano per il calcolo della global inequality sono numerosi, sia di natura empirica sia di natura concettuale. La possibilità di misurare empiricamente la "diseguaglianza globale" nella sua terza accezione è relativamente recente. La sua analisi richiede di disporre non solo di dati relativi ai redditi medi dei diversi paesi tra loro comparabili, ma anche di indagini campionarie che consentano di ricostruire la funzione che rappresenta la distribuzione personale dei redditi tra $\mathrm{i}$ "cittadini del mondo", come se appartenessero ad una sola entità territoriale. E' proprio questa distribuzione, che richiede la comparabilità tra valori provenienti da fonti diverse, la più difficile da stimare. Un considerevole sforzo è stato diretto, negli ultimi anni, a costruire banche dati contenenti informazioni armonizzate e comparabili. Esempi di queste iniziative sono i data base costruiti nell'ambito del "Luxembourg Income Study" (LIS) e del "Wealth Study" (LWS) che riguardano molti paesi OECD, dallo WIDER, e dal "Living Standards Measurement Survey" (LSMS) condotto all'interno della Banca Mondiale (Milanovic, 2006b). Tuttavia il numero dei paesi presi in considerazione per questo processo di omogeneizzazione è piccolo rispetto al numero di paesi esistenti.

La funzione di distribuzione dei redditi individuali a livello mondiale può essere stimata seguendo due diverse metodologie. Un primo gruppo di lavori fa uso delle indagini campionarie solo per ottenere informazioni sulle quote di reddito spettanti ai "quantili" nei quali è stata suddivisa la popolazione. Il valore dei livelli medi dei redditi (o dei consumi) sono imputati sulla base dei valori desunti dalla contabilità nazionale. Un secondo gruppo di lavori, invece, fa ricorso esclusivamente alle indagini campionarie sui redditi familiari, sia per il calcolo della diseguaglianza che per il calcolo dei livelli medi delle variabili economiche di interesse. In questi studi la distribuzione globale è ottenuta direttamente dalle indagini campionarie per un numero molto elevato di paesi. In base alla funzione di distribuzione stimata viene, successivamente calcolato un indice "tradizionale" di diseguaglianza come l'indice di Gini o l'indice di Theil. 
Le stime fornite da Milanovic (2012), riportate nella Fig. 1, consentono di evidenziare le differenze nella dinamica della diseguaglianza globale, nel periodo successivo alla seconda guerra mondiale, in relazione alla specifica misura adottata, e cioè a seconda che ci si riferisca alla "diseguaglianza tra paesi", alla diseguaglianza internazionale" o, infine, alla "diseguaglianza globale". Sull'asse orizzontale sono indicati gli anni. Sull'asse verticale è riportato come misura della diseguaglianza l'indice di Gini. La composizione del campione di paesi considerati è rimasta praticamente costante a partire dal 1960.

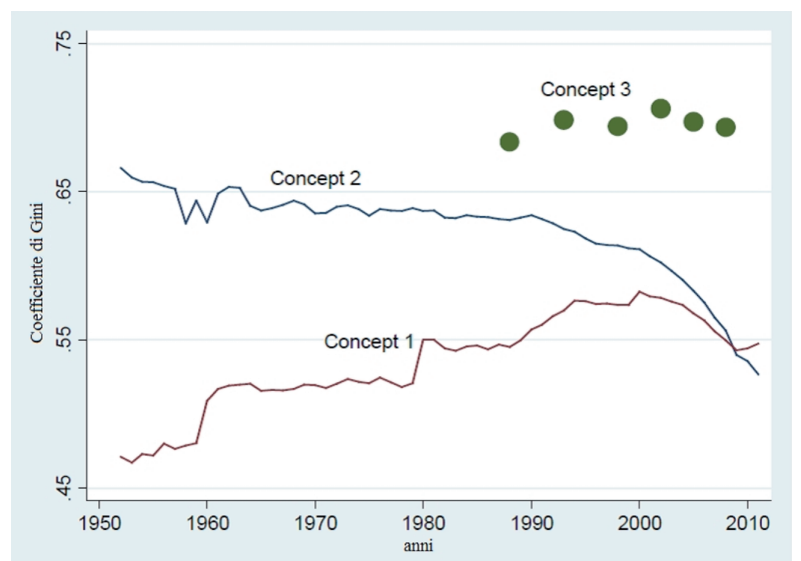

Fonte: Milanovic (2012, p. 6). Valori calcolati con i nuovi prezzi a parità di potere d'acquisto.

Fig. 1 - Indici di diseguaglianza "tra paesi", "internazionale" e "globale", 1952-2011.

L'indice della diseguaglianza "tra paesi" (Concept 1) calcolato sulla base di redditi medi "a parità di potere d'acquisto", e quando questi non siano pesati per la numerosità della popolazione è crescente nel periodo 1950-1960 e tra il 1980 ed il 2000. Si osserva una certa stabilità attorno ad un valore di 53 tra il 1960 ed il 1980. A partire dall'inizio degli anni 80, in coincidenza con la crescita dei tassi d'interesse reale e con l'emergere della crisi debitoria in molti paesi in via di sviluppo si è verificato un processo di significativa divergenza tra i redditi pro-capite dei diversi paesi e dunque di crescita dell'indice di diseguaglianza. La cosiddetta "decade perduta" in America Latina, la stagnazione e poi il vero e proprio declino nei paesi dell'Europa dell'Est e nei paesi dell'ex Unione Sovietica, ed il pessimo andamento di molti paesi africani sono stati i fattori che, insieme all'ottimo andamento dei paesi ricchi spiega- 
no la divergenza. In questo periodo di crescente globalizzazione i paesi ricchi sono cresciuti più velocemente di quelli poveri.

A partire dal 2001 la diseguaglianza ha cessato di crescere, e il trend si è invertito. Nonostante l'inversione nella tendenza della diseguaglianza, il suo livello è, oggi, in misura significativa maggiore di quanto non fosse negli anni 60 e 70 . Il periodo compreso tra il 2001 ed il 2006 è stato di crescita non solo per l'economia globale (trainata dai paesi maggiori e più ricchi), ma anche per i paesi africani, per i paesi excomunisti e per quelli dell'America Latina. Il tasso di crescita di questi paesi spiega l'inversione nella tendenza dell'indice dopo il 2001.

Il primo concetto di diseguaglianza globale, quello che misura la "diseguaglianza tra paesi", è l'indice favorito in macroeconomia. E' questa l'accezione del concetto più appropriata quando si voglia verificare l'ipotesi della convergenza/divergenza nei livelli di reddito tra $\mathrm{i}$ diversi paesi. Le prime analisi, di natura empirica si collocano all'interno degli studi sulla crescita. Questi studi vertono sul tema della convergenza ovvero della divergenza tra i redditi procapite dei paesi in via di sviluppo rispetto a quelli industrializzati. Più precisamente ci si riferisce al tentativo di verificare l'ipotesi, così come prevista dal modello neoclassico nella versione di Solow (1956), della convergenza condizionale. Convergenza perché, se tale ipotesi fosse verificata, i paesi poveri, prima o poi, convergerebbero allo stesso reddito pro capite di quelli ricchi. Condizionale perché vera a condizione che alcuni dei parametri che caratterizzano le diverse economie, ed in particolare il tasso di risparmio e il rapporto capitale prodotto, siano i medesimi.

L'analisi della dinamica della diseguaglianza "internazionale" (Concept 2) può essere effettuata, anche, sulla base di due diversi campioni, uno che include ed uno che esclude la Cina (Milanovic, 2009). Quando si includa la Cina, l'indice è andato diminuendo negli ultimi 60 anni, con una accelerazione a partire dal 2000. Il valore dell'indice nel 1960 era molto elevato pari a 0.65 ed alla fine del 2006 l'indice si era ridotto allo 0.55 (Fig. 2). Se, invece, si calcola l'indice escludendo la Cina si nota una sua crescita tra il 1952 ed il 2000. In quell'anno si verifica un'inversione con un declino tra il 2000 ed il 2010. La diminuzione dell'indice, come evidenzia la Fig. 2, è più accelerata nel caso si includa la Cina. In Cina, infatti, il reddito medio pro capite è aumentato, a partire dal 1980 da un livello di reddito molto basso non solo più della media mondiale, ma anche in misura superiore rispetto ai paesi ricchi, ed in particolare rispetto agli Stati Uniti. Nel periodo 
successivo al 2000 un'importante stimolo è derivato anche dalla crescita dell'India. Per questa ragione l'indice decresce anche quando si escluda la Cina.

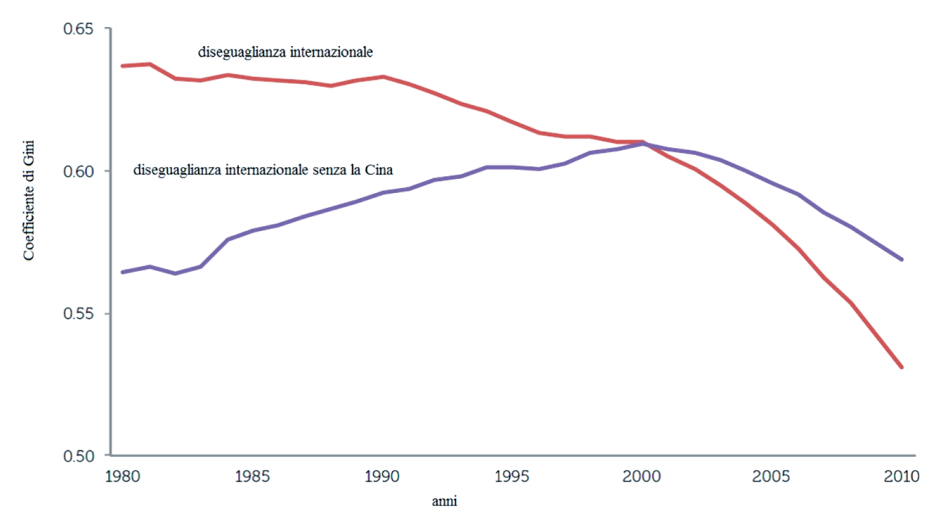

Fonte: Milanovic (2009, p. 9).

Fig. 2 - La diseguaglianza internazionale "senza" e con la Cina, 1980-2010.

Coloro che ritengono che gli effetti positivi della globalizzazione siano stati molto numerosi sottolineano come, i divari di reddito pesati per la popolazione, e dunque misurati con l'International inequality, siano diminuiti. Coloro che, invece, sono critici nei confronti della globalizzazione preferiscono considerare l'Intercountry inequality come misura della crescente divergenza nei redditi pro-capite dei diversi paesi. Secondo Bourguignon (2011, p. 5), d'altra parte "Proponents of the view that, based on this kind of evidence, inequality in the world has continued to increase in the recent past are not completely wrong. They are simply using an inequality measure that is somewhat extreme in the sense that what matters in measuring inequality is the relative level of welfare of the poorest individuals in the world compared to the richest, irrespectively of how many they are. In other words, the fact that a few bundred millions of people have escaped poverty in China, India, Bangladesh and other large countries cannot compensate the fact that 3 millions of Burundians have on average become slightly poorer".

L'utilizzo dell'indice di Gini per stimare la "diseguaglianza globale" merita un chiarimento di natura concettuale. L'indice di Gini ha non solo il significato statistico di misura della concentrazione della distribuzione dei redditi, ma gli può essere attribuito anche un significato 
normativo. Essendo derivato dalla curva di concentrazione di Lorenz questo indice misura il senso di privazione relativa che ciascun percettore prova nel confrontare la propria posizione reddituale con quella di tutti gli altri. Potrebbe essere difficile ipotizzare che questo significato possa essere preservato quando il confronto avviene tra cittadini che vivono in paesi così distanti tra loro come l'Africa sub-sahariana rispetto al Canada o agli Stati Uniti. Sicuramente è stato così in passato. Oggi, tuttavia, proprio la globalizzazione e la progressiva integrazione internazionale ha modificato la percezione della propria posizione reddituale relativa nel confronto con gli altri cittadini del mondo. L'ipotesi che il confronto tra redditi individuali avvenga non solo all'interno dei confini nazionali, ma anche all'esterno di questi, sembra essere divenuta oggi non solo accettabile, ma addirittura esplicativa di importanti fenomeni connessi alla globalizzazione (Milanovic, 2006b, p. 149). L'accelerazione dei flussi migratori che si è verificata negli ultimi anni è certamente uno di questi.

Al dibattito sulle tendenze della "diseguaglianza globale" nelle sua terza accezione, hanno contribuito numerosi autori a partire dal lavoro pionieristico di Sala-I-Martin, (2002). Sembra esservi un accordo circa la dimensione mentre manca il consenso sulle tendenze più recenti. L'accordo sul valore risulta particolarmente significativo visto che le metodologie di stima sono alquanto differenti. I valori dell'indice di Gini, con due eccezioni estreme, cadono in un intervallo molto ristretto tra lo 0.63 e lo 0.68 (Milanovic, 2006b, p. 140). Milanovic, utilizzando $i$ dati disponibili più recenti "a giant accumulation of individual survey data adjusted by country-specific PPP Exchange rates" (Milanovic, 2011, p. 11), ha calcolato il valore della diseguaglianza globale misurata dal coefficiente di Gini per alcuni anni successivi al 1993 (Figg. 1, 3). L'indice presenta un trend ciclico: dopo avere registrato un valore sostanzialmente stabile - attorno a 0.70 - nel periodo 1990-2005, diminuisce lievemente raggiungendo un valore di 0.670.68 nel 2010. Questi mutamenti sono spiegati nei primi anni 90 dalla crescita lenta dei redditi nelle zone rurali di India e Cina e dal collasso dell'Europa dell'Est. Entrambi questi fattori hanno contribuito alla crescita della diseguaglianza globale. Quando entrambe queste tendenze si sono invertite nel quinquennio successivo la diseguaglianza globale è diminuita. Queste sono inversioni di tendenza causate da specifici eventi economici in paesi grandi, non una tendenza. 


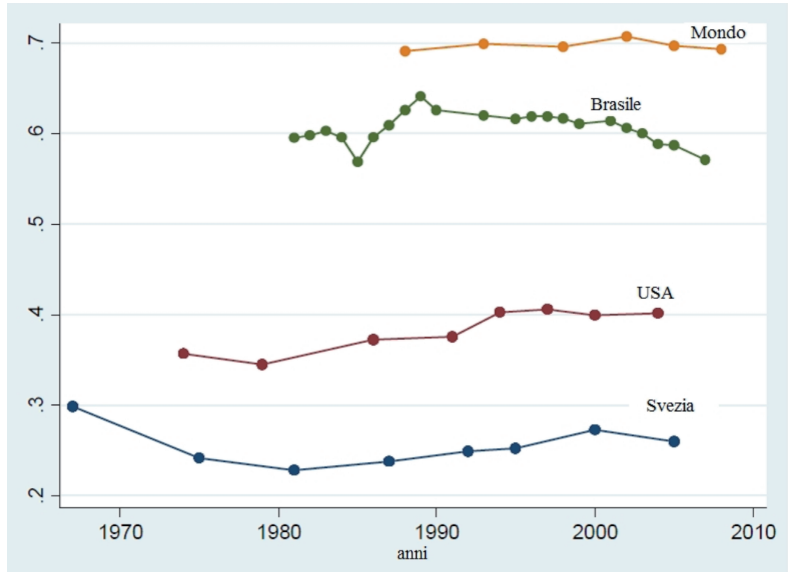

Fonti: Milanovic (2012, p. 9). L'indice di Gini è calcolato sui redditi disponibili in base ai "World Income Distribution database".

Fig. 3 - Che cosa significa un indice di Gini pari a 0.70 ?

L'indice di Gini presenta comunque sempre un valore significativamente superiore a quello che si riscontra all'interno di qualsiasi altro paese, compresi quelli (Sud Africa e Brasile) caratterizzati da una diseguaglianza particolarmente elevata (Fig. 3). Si osservi che un valore di Gini pari a circa 0,70 comporta che l'1 per cento della popolazione mondiale più ricco riceve quasi il 14 percento del reddito globale, mentre il 20 per cento più povero riceve solo l'1 per cento.

Il lavoro di Bourguignon e Morrisson (2002) è stato uno dei primi ambiziosi tentativi di ricostruire l'andamento della diseguaglianza nel lungo periodo tra il 1820 ed il 1990, utilizzando sia dati sui redditi pro capite desunti da fonti di contabilità nazionale sia dati sulle quote distribuite ai diversi gruppi di percettori desunti da una pluralità di fonti micro. I risultati ottenuti dai due autori sono molto eloquenti: la diseguaglianza tra i redditi dei cittadini del mondo, misurata dall'indice di Gini, ha registrato un drastico aumento nel periodo fra il 1820 e il 1950 circa, mentre successivamente il suo tasso di crescita è sensibilmente diminuito fino ad arrestarsi nell'ultimo periodo.

Sempre Bourguignon (2011) calcola l'evoluzione della diseguaglianza globale dal 1910 al 2010 utilizzando due diverse misure della diseguaglianza e cioè sia l'indice di Gini, sia il rapporto tra la quota di reddito del decile più ricco rispetto a quello più povero (Fig. 4). 
Entrambi i quantili comprendono individui appartenenti sia a paesi ricchi sia a paesi poveri. I due periodi 1910-1989 e 1989-2010 non sono comparabili. In corrispondenza al 1989 si osserva un'interruzione nella serie storica determinata dal mutamento del campione. Nel primo periodo il campione conteneva un numero inferiore di paesi. Inoltre nei due periodi i valori reddituali sono stati resi comprabili utilizzando parità di potere d'acquisto differenti (Bourguignon, 2013, p. 5). Sulle ordinate sono indicati: a sinistra l'indice di Gini e a destra il rapporto tra il reddito del $10 \%$ più ricco rispetto al $10 \%$ più povero.

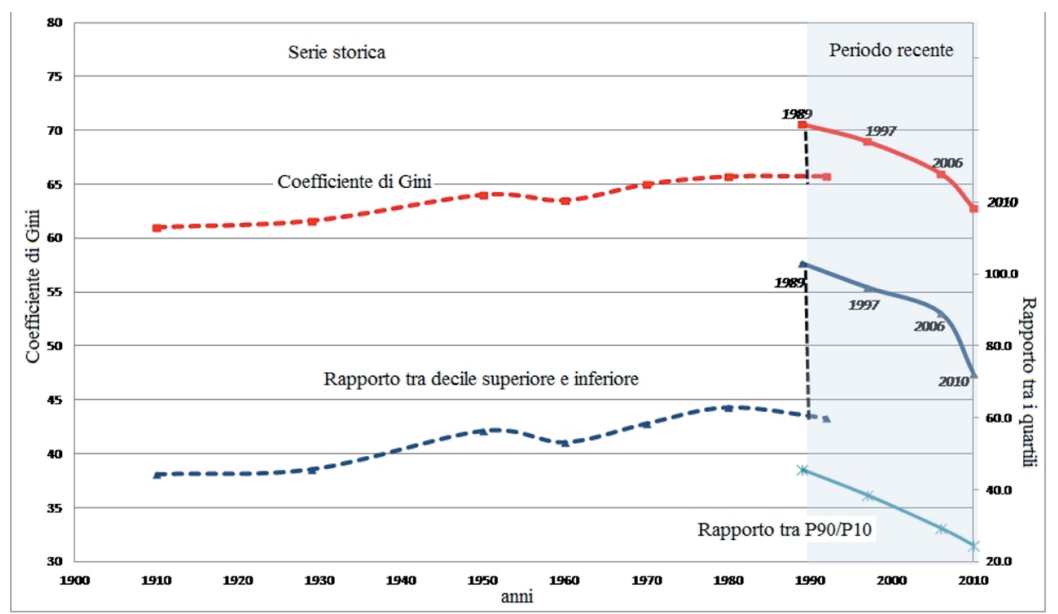

Fonte: Bourguignon (2013, p. 5).

Fig. 4 - L'andamento della "diseguaglianza globale", 1910-2010 (varie misure).

Entrambi gli indici sono crescenti fino al 1989, ad eccezione di una lieve diminuzione tra il 1950 ed 1960. La diminuzione appare anche tra il 1980 ed il 1990 se si considera l'indice ottenuto come rapporto tra i due quantili di reddito. A partire da quest'anno la tendenza alla crescita si inverte per entrambi gli indici. La diseguaglianza globale diminuisce ad un ritmo molto rapido. Bourguignon $(2011$, p. 1) sottolinea come ci si trovi "at a turning point in history. A secular trend of increasing inequality is being reversed. Inequality in the world has continuously increased for a century and a half since the beginning of the Industrial Revolution. It is now decreasing at the same time as global growth is accelerating.....Seeing as inequality seems to be increasing in a number of countries, could it be the case that domestic inequality will replace international inequality to 
some extent? If the rise of domestic inequalities were to be somehow associated with globalization in the public opinion, could it be a threat to the process of global development as a whole?".

Anche l'indice stimato da Milanovic, e riportato nella Fig. 3, segnala una diminuzione a partire dal 2000 rispetto al periodo precedente di relativa stabilità. L'ipotesi è che la riduzione nella diseguaglianza globale sia da attribuirsi alla crescita dei paesi in via di sviluppo che è stata più rapida di quella dei paesi industrializzati. Secondo Bourguignon (2011, p. 3) "there seems to have been a growth rate gap between the North and the South over the last 10 years or so, the width of which has not changed significantly in the last 5 years. If this trend continues, it certainly is an important novelty in the history of global economy".

Una delle domande che sorge osservando la Fig. 1 è come sia possibile conciliare la tendenza dell'indice che misura la "diseguaglianza internazionale", costante nel periodo 1980-1990 e decrescente nel periodo successivo, con la dinamica dell'indice che misura la diseguaglianza globale che, nello stesso periodo, è stata, sia pure lievemente, crescente. Per comprendere questo "puzzle" è necessario considerare non solo la dinamica della diseguaglianza tra paesi, ma anche di quella all'interno dei singoli paesi. La diseguaglianza globale può essere considerata come la risultante di entrambe le diseguaglianze, quella between e quella within. La prima è misurata dai divari nei redditi medi dei diversi paesi. La seconda, invece, è misurata come divario tra i singoli redditi individuali all'interno dei paesi. Nel calcolo dell'indice della "diseguaglianza internazionale" ad ogni cittadino viene assegnato il reddito medio del paese d'appartenenza, nel calcolo dell'indice della "diseguaglianza globale", invece, ad ogni cittadino è assegnato il proprio reddito. I redditi medi dei paesi in via di sviluppo hanno iniziato a convergere verso quelli dei paesi ricchi. Invece i redditi dei diversi gruppi di percettori, all'interno dei singoli paesi, non sono cresciuti in modo da convergere. E' così possibile osservare un processo di convergenza tra paesi, ma di divergenza tra cittadini all'interno dei singoli paesi.

La Fig. 5 mostra l'andamento della diseguaglianza globale misurata dall'indice di Theil, e l'andamento dei due indici che lo compongono, ovvero della diseguaglianza within e between. L'indice di Theil, nel lungo periodo, ha un andamento molto simile a quello di Gini riportato nella Fig. 4. La diseguaglianza tra paesi, una delle due componenti della diseguaglianza globale, rivela un profilo molto simile a quello della diseguaglianza globale con una crescita fino al 1950 seguito da un periodo 
di relativa stabilità ed una netta diminuzione dopo il 1989. La diseguaglianza all'interno dei paesi, mostra, invece una relativa stabilità fino all'inizio del 900, una diminuzione tra il 1900 ed il 1950, ed infine un lieve aumento nei decenni successivi. Questo aumento, nell'ultimo decennio, è stato compensato dalla diminuzione della diseguaglianza tra paesi, così che la diseguaglianza globale è diminuita. Si è già osservato come tale riduzione sia stata "trainata" dall'aumento del reddito medio di paesi popolosi come la Cina e l'India.

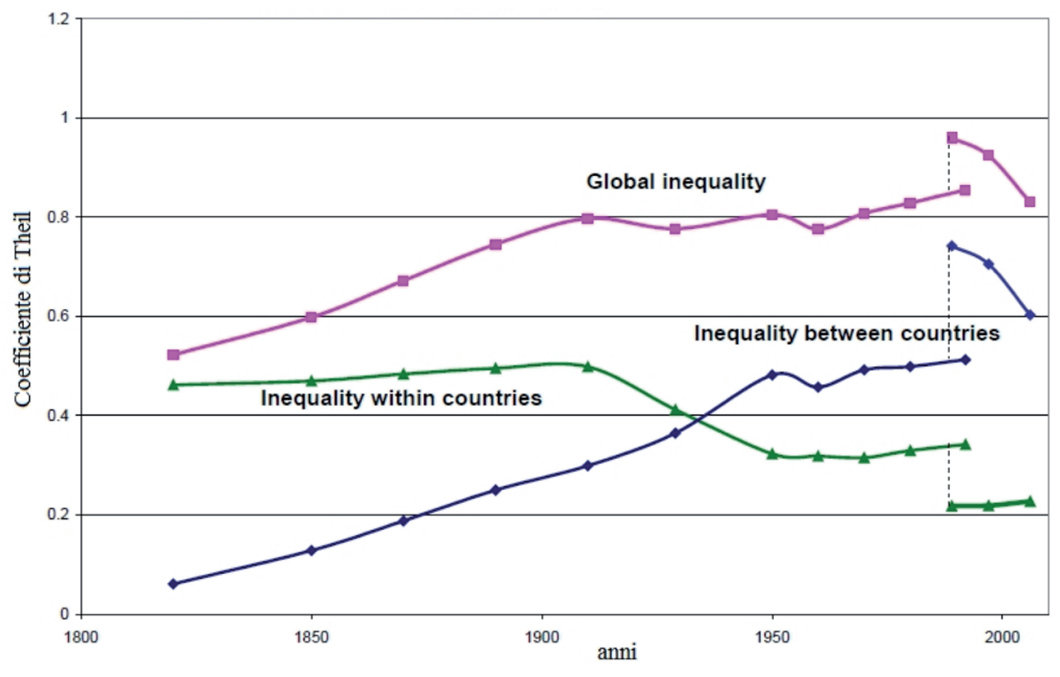

Fonte: Bourguignon (2012, p. 7).

Fig. 5 - Scomposizione della diseguaglianza globale nelle due componenti "within" e "between" (Coefficiente di Theil).

Una seconda domanda che sorge è quale possa essere il significato del mutamento verificatosi nel peso tra le due componenti within e between. La diseguaglianza interna può essere interpretata come la componente attribuibile alle differenze nei redditi tra classi di percettori all'interno d'ogni paese. La diseguaglianza between, invece, e cioè quella tra i redditi medi dei diversi paesi può essere interpretata come la componente della diseguaglianza globale che dipende dalla "localizzazione" degli stessi paesi. Seguendo Milanovic (2012) si può osservare la diseguaglianza si è modificata nelle sue due componenti passando da un valore attribuibile prevalentemente alle differenze di classe "interne" ad ogni paese ad un valore collegato principalmente ai divari di reddito "tra paesi". 
La Fig. 6 evidenzia le differenze nella composizione dell'indice di diseguaglianza di Theil nel 1870 e nel 2000, scomposto nelle due componenti: within e between. La "cittadinanza", e dunque le differenze tra paesi, spiegherebbero oggi poco più del 60 per cento della diseguaglianza globale. Si tratta di un mutamento significativo rispetto al passato, quando era la diseguaglianza within a pesare maggiormente. Questo significa che se il reddito personale dipende in larga misura dalla cittadinanza si può affermare che non esiste eguaglianza di opportunità a livello globale e che la cittadinanza costituisce una vera propria rendita non dipendente dagli sforzi individuali (Milanovic, 2012, p. 20).

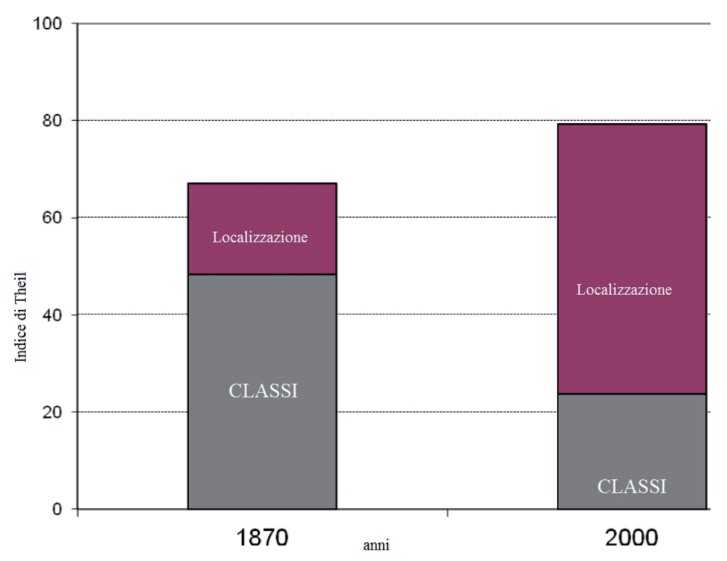

Fonte: Milanovic (2012, p. 20).

Fig. 6 - Livello e composizione della diseguaglianza globale nel $19^{\circ}$ secolo ed attorno al 2000 misurata dall'Indice di Theil.

Naturalmente molti altri fattori determinano il livello di reddito. Tuttavia "the remarkable thing is that a very large chunk of our income will be determined by only one variable, citizenship, that we, generally, acquire at birth. It is almost the same as saying, that it is possible predict ber income just from the knowledge of her citizenship". Anche Raymond Aron (1961, p. 60) in The Dawn of Universal History afferma che "In a buman society in the process of unification inequality between nations acquires the same meaning as inequality among classes in the past. Standards of living differ today between continents or between countries more than they ever did. At the same time, the perception of inequality increases whereas resignation to poverty and to destiny is disappearing". 


\section{CHI HA BENEFICIATO DEI MUTAMENTI DELLA DISEGUAGLIANZA GLOBALE?}

Le stime fornite da Milanovic (2012) consentono di identificare le classi di percettori che hanno tratto vantaggio dai mutamenti nella disuguaglianza globale. Il confronto tra le due curve di Lorenz stimate per il 1988 e per il 2008 consente di osservare se vi sia stata o meno una diminuzione della diseguaglianza nella distribuzione globale dei redditi. Com'è noto l'asse verticale riporta le percentuali cumulate di reddito possedute dai diversi quantili di popolazione, le cui percentuali cumulate sono indicate sull'asse orizzontale. Quanto più la curva di Lorenz è vicina alla retta di equidistribuzione (collocata con un angolo a 45 gradi), tanto più significa che la diseguaglianza è bassa. I dati riportati nella Fig. 7 mostrano che nessuna delle due curve domina nel senso di Lorenz, e cioè corrisponde ad una maggiore equidistribuzione per tutto l'intervallo di valori. La curva corrispondente alla distribuzione del 2008 è più vicina alla retta di equidistribuzione, e quindi domina la curva del 1988 , negli intervalli intermedi fino all' $80^{\circ}$ percentile. Si è verificato, dunque, un arricchimento dei percettori che appartengono ai gruppi più ricchi. Per i gruppi più poveri i redditi sono rimasti stagnanti.

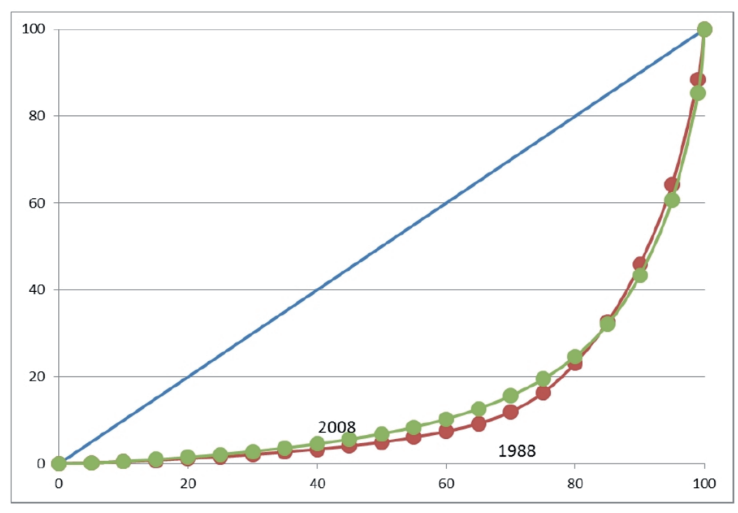

Fonte: Milanovic (2012, p. 16).

Fig. 7 - Curve di Lorenz.

Il mutamento nel livello di diseguaglianza appare ancora più evidente quando si consideri la distribuzione del reddito nei diversi quantili di popolazione. La Fig. 8 evidenzia il mutamento del reddito percepito dai vari quantili di popolazione tra il 1988 ed il 1998. Due appaiono i gruppi che 
hanno beneficiato della crescita mondiale del reddito e che si possono considerare i principali "vincitori" della globalizzazione: i percettori più ricchi e coloro che appartengono alla classe media in paesi in via di sviluppo come la Cina, l'India, l'Indonesia ed il Brasile. Il reddito reale dell'1\% più ricco è cresciuto più del $60 \%$ nelle ultime due decadi. D'altra parte si sono verificati, anche, aumenti significativi attorno alla mediana. "It is there, between the 50th and 60th percentile of the global income distribution that we find some 200 million Chinese, 90 million Indians, and about 30 million people each from Indonesia, Brazil and Egypt" (Milanovic, 2012, p. 11).

Anche coloro che si collocano in corrispondenza ai primi tre percentili hanno ottenuto guadagni significativi. I redditi di questi gruppi in termini reali sono cresciuti tra il $40 \%$ ed il $70 \%$. La sola eccezione è costituita dal $5 \%$ più povero i cui redditi in termini reali sono rimasti praticamente invariati. I gruppi compresi tra il 65 ed il 75 simo percentile sono da considerarsi il gruppo perdente. Questi percettori che possono essere definiti come una "global upper-middle class" includono cittadini dei paesi industrializzati, degli ex-paesi sovietici e dell'America Latina che non hanno sperimentato alcuna crescita nei loro redditi. In sintesi si può affermare che a livello globale si stia formando una classe media soprattutto grazie al contributo di alcuni paesi in via di sviluppo particolarmente dinamici. Nello stesso tempo è aumentata la polarizzazione dei redditi a causa delle crescita più rapida dei redditi più elevati.

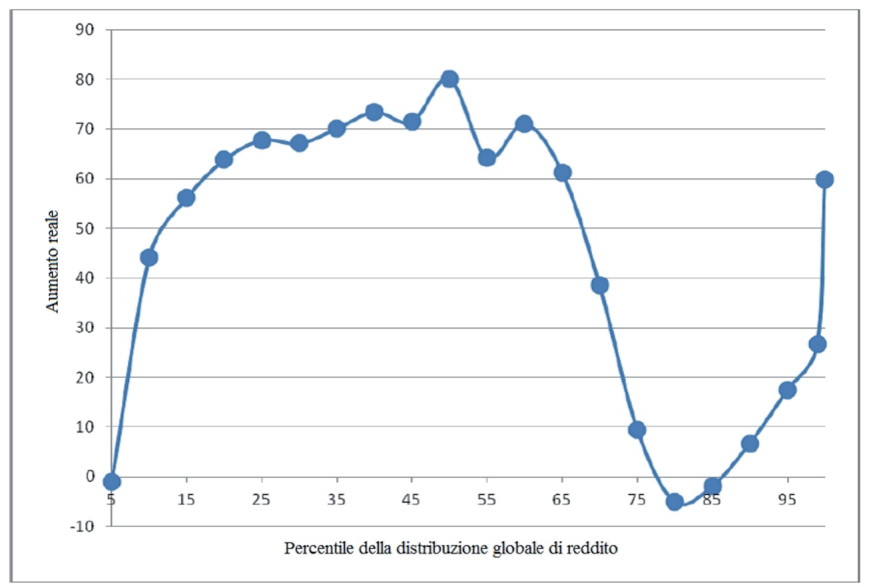

Fonte: Milanovic (2012, p. 11).

Fig. 8 - Mutamenti nel reddito reale tra il 1988 ed il 2008 in corrispondenza a diversi percentile della distribuzione globale del reddito (calcolati in dollari 2005). 
I mutamenti osservati sono la risultante di quanto è accaduto all'interno dei vari paesi in termini di crescita della disuguaglianza. E' possibile individuare i differenti meccanismi che determinano il livello e la dinamica della diseguaglianza, nei paesi industrializzati ed in quelli in via di sviluppo, distinguendo tra fattori endogeni ed esogeni, e cioè quelli riconducibili al processo di globalizzazione. Nei paesi industrializzati fattori come la concentrazione della ricchezza, il peso relativo dei redditi da capitale rispetto a quelli da lavoro, l'ineguale accesso all'educazione, il dualismo territoriale, fattori demografici e politiche redistributive, devono essere tutti ritenuti all'origine della diseguaglianza. Tuttavia, da soli, non riescono a spiegare la sua crescita negli ultimi decenni. Altri fattori esogeni che si suppone interessino tutte le economie industrializzate e che prevalgano, nel lungo periodo, sulle specificità nazionali hanno contribuito ad accrescere i divari distributivi.

Il processo di apertura e di liberalizzazione dei mercati nazionali ed internazionali conseguente all'intensificarsi della globalizzazione, l'adozione di tecnologie risparmiatrici di lavoro come le Information, Communication Technology (ICT), la necessità di adeguare i contesti nazionali alla accresciuta competizione con gli altri paesi, in particolare con quelli in via di sviluppo, avrebbero prodotto un mutamento nelle strutture produttive, ridotto l'occupazione, il potere contrattuale dei lavoratori. e dunque anche della quota dei redditi da lavoro. Anche l'accresciuta mobilità dei capitali, conseguente alla progressiva integrazione dei mercati finanziari che ha caratterizzato il processo di globalizzazione si è tradotta in una crescita dei profitti, dei redditi più elevati e dunque anche della diseguaglianza (OECD, 2011a, 2013).

Un fenomeno che ha recentemente attirato l'attenzione degli studiosi, e in particolare di Atkinson, Piketty e Saez (2007) è la crescita dei redditi molto elevati, che ha determinato un aumento della quota di reddito percepita dal percentile più ricco. L'evidenza empirica fornisce supporto all'ipotesi secondo cui l'internazionalizzazione dei mercati, anche di quelli del lavoro, avrebbe favorito l'aumento della diseguaglianza all'interno dei paesi. Nella maggior parte dei paesi, una quota significativa di questo aumento è attribuibile all'aumento dei "top labor incomes, and especially wages and salaries" (Atkinson et al., 2011, p. 5). il funzionamento del mercato internazionale per $\mathrm{i}$ "managers and for superstars" viene considerato all'origine degli aumenti. I top incomes percepiti corrispondono ad una percentuale molto ridotta di percettori ma ad una quota significativa del reddito nazionale e del totale delle 
entrate fiscali. Sia il tasso di crescita del reddito nazionale che l'indice di Gini assumono di conseguenza valori differenti a seconda che i redditi più elevati siano esclusi dalle stime o vi siano compresi.

Un terzo fattore considerato all'origine della diminuzione relativa della quota di reddito percepita dal lavoro è costituito dai mutamenti nei meccanismi istituzionali che regolano, nei vari paesi, il funzionamento del mercato del lavoro, ossia la riduzione della regolamentazione, l'erosione del salario minimo e del potere sindacale, l' incremento della mobilità (Krugman, 2007; Glyn, 2009). Questi mutamenti hanno accentuato le spinte verso la diseguaglianza. Nel contempo le riforme rese necessarie dall'aumentata competizione internazionale hanno contribuito alla riduzione della quota di reddito affluita al lavoro dipendente. Le prospettive economiche dei lavoratori poco qualificati dei settori tradizionali sono state compromesse anche dal trasferimento verso i paesi in via di sviluppo delle fasi più tradizionali e a più basso contenuto tecnologico della filiera produttiva. In seguito al diffondersi dell'outsourcing si sarebbe verificata, da parte delle imprese dei paesi industrializzati, una riduzione della domanda di lavoratori poco qualificati: ne sarebbe seguito un ampliamento dei ventagli retributivi all'interno della categoria dei lavoratori dipendenti (Krugman, 2007).

I fattori endogeni responsabili della crescita della diseguaglianza nei paesi in via di sviluppo sono differenti rispetto a quelli che operano nei paesi industrializzati (OECD, 2011b). Tra i più importanti è necessario annoverare l'esistenza di un ampio settore informale, un significativo divario tra settore urbano e settore rurale, bassi livelli di istruzione per ampie fasce di popolazione, discriminazione di genere e barriere all'accesso sul mercato del lavoro per le donne. Tra i fattori esogeni, anche in questo caso, occorre segnalare l'importanza della globalizzazione che, attraverso gli incentivi alla produzione e alle esportazioni, finisce con il favorire solo alcune zone o settori di un paese, accentuando i divari regionali e quelli nelle tecnologie adottate. Circa il 60\% dei PVS mostra una crescita della diseguaglianza nell'ultimo decennio. Solo una minoranza di paesi, come quelli dell'America Latina, hanno sperimentato, invece, una diminuzione.

\section{DA “PROLETARI” A MIGRANTI}

Al fine di evidenziare le differenze nella posizione reddituale di individui che abitano in paesi diversi, la popolazione di tutti i paesi è 
stata suddivisa in gruppi comprendenti il 5\% (ventili) dei rispettivi percettori ordinati dai più poveri ai più ricchi. I ventili sono rappresentati sull'asse orizzontale della Fig. 9. Sull'asse verticale sono riportati i percentili della distribuzione del reddito di tutti i paesi del mondo ottenuta ordinando in senso non decrescente i redditi medi dei ventili di reddito di ogni paese (Milanovic, 2011). In questo modo, il reddito medio dei ventili di ciascun paese, corrisponde ad uno dei percentili di reddito globale collocati sull'asse verticale. Questi percentili sono stati quindi ottenuti partendo dalla distribuzione globale dei redditi medi di ogni ventile indipendentemente dal paese di appartenenza.

In altri termini, se $\mathrm{N}$ sono i paesi del mondo considerati, i percentili della distribuzione del reddito mondiale sono stati determinati sulla base dei redditi medi degli Nx20 ventili di popolazione Con riferimento, ad esempio, agli Stati Uniti, il reddito medio del ventile più povero si colloca al $60^{\circ}$ percentile della distribuzione del reddito mondiale. Sul versante opposto, il $5 \%$ di americani più ricco si colloca in corrispondenza all' $1 \%$ più ricco del mondo.

Se si prende in considerazione un altro paese come l'India (il paese più povero di quelli rappresentati in Fig. 9), si osserva che essa raggiunge il 60-esimo percentile (e, quindi, la posizione del $5 \%$ più povero degli Stati Uniti) solo a partire dal 20-esimo ventile. Quindi, anche se sicuramente in questo paese alcuni individui sono molto ricchi, il reddito medio del ventile più ricco, comunque non si colloca al di sopra del $70^{\circ}$ percentile della distribuzione mondiale di reddito. Il numero di cittadini indiani che hanno redditi (Milanovic, 2012, p. 23) comparabili a quelli della classe media americana è dunque ancora molto limitato. Se si fissa l'attenzione sulla Russia (il paese che occupa la seconda posizione) la Fig. 9 indica che il $5 \%$ più povero si colloca intorno al 38-esimo percentile e che il 60-esimo percentile viene superato solo a partire dal quarto ventile.

La distribuzione del reddito in Cina domina quella indiana per tutto l'intervallo di valori posti sull'asse orizzontale. Questo significa, che individui che si collocano "at a given percentile level of Chinese distribution always have higher income than people at that same percentile of India's income distribution" e che il ventile di percettori più ricco, in Cina, si colloca praticamente all' $80^{\circ}$ percentile della distribuzione mondiale del reddito. "If we used percentiles, the top 1\% of the Chinese would be better-off than 93\% of world population" (Milanovic, 2012, p. 23). Nel caso del Brasile il gruppo dei più poveri si colloca nella parte 
inferiore della distribuzione mondiale del reddito. Esiste, invece, una consistente classe media che si colloca tra il $70^{\circ}$ e $80^{\circ}$ percentile del reddito mondiale. Allo stesso modo i brasiliani più ricchi si collocano in corrispondenza ai percentili superiori.

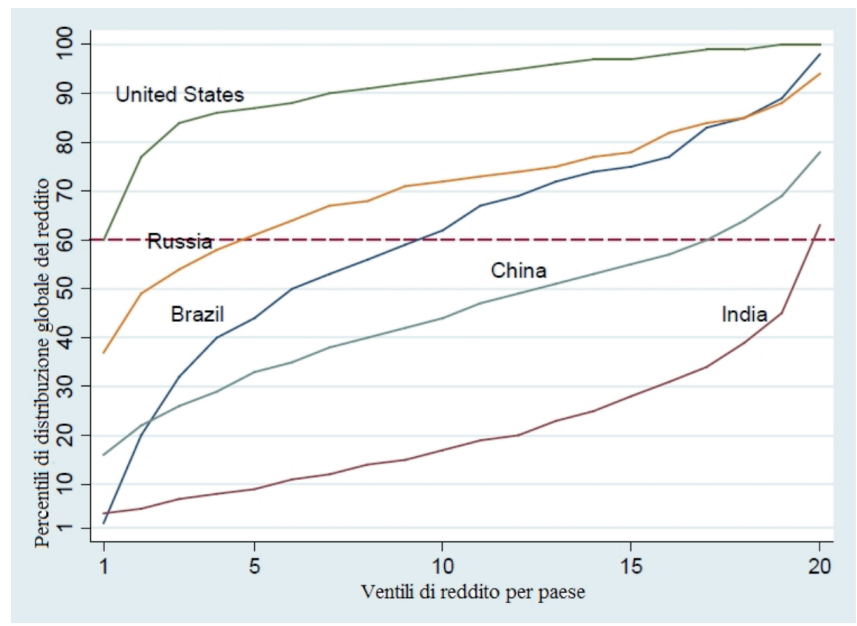

Fonte: Milanovic (2011, p. 8).

Fig. 9 - Paesi diversi e classi di reddito nella distribuzione globale del reddito, 2005. (La linea all'altezza del $60^{\circ}$ percentile corrisponde al $5 \%$ più povero della popolazione americana).

La Fig. 10 evidenzia le differenze nella distribuzione del reddito dei ventili in Italia, considerato come paese di arrivo per i flussi migratori, e nei paesi vicini dai quali i flussi provengono in larga misura. Il confronto potrebbe essere, naturalmente, effettuato anche con il resto del mondo e con altri paesi europei. I gruppi più poveri in Italia si collocano immediatamente al di sotto del $60^{\circ}$ percentile, in modo del tutto analogo a quanto avviene negli Stati Uniti. Nello stesso tempo il 5\% degli italiani più ricchi si colloca in corrispondenza ai percentili superiori. I cittadini poveri di paesi come la Germania, o potrebbero essere anche di altri paesi nordici, invece si collocano in corrispondenza all' $80^{\circ}$ percentile del reddito mondiale.

In Albania, ad esempio, circa il 30\% della popolazione dispone di un reddito il cui valore si colloca sotto la linea della povertà italiana. Questo significa che se questo gruppo di popolazione emigrasse in Italia, se anche ricevesse un reddito pari a quello dei più poveri italiani, 
si collocherebbe comunque ad un livello di reddito comunque superiore a quello che avrebbe ottenuto rimanendo in Albania. Anche in Argentina, sempre a titolo d'esempio, circa un quarto della popolazione percepisce un reddito tale da collocarla al di sotto della linea della povertà italiana. Nei paesi africani, infine, come ad esempio la Costa d'Avorio, i ventili più ricchi superano di poco il $65^{\circ}$ percentile. Questo significa che i più ricchi in Costa d'Avorio hanno una posizione reddittuale simile a quella dei più poveri in Italia o in Germania. In questo paese circa l' $80 \%$ della popolazione si colloca sotto la linea della povertà italiana. Emigrando questi cittadini avrebbero, dunque, la probabilità di migliorare la propria posizione.

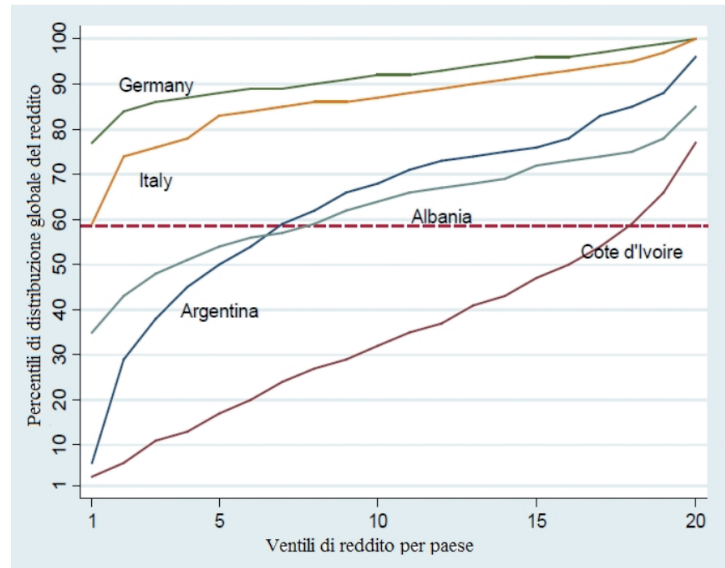

Fonte: Milanovic (2012, p. 24).

Fig. 10 - Italia e altri paesi.

\section{CONSIDERAZIONI CONCLUSIVE}

L'evidenza empirica che negli ultimi anni è stata resa disponibile non fornisce ancora risultati concordi. Le interpretazioni che se ne possono trarre sono difficilmente generalizzabili, in relazione alle differenze nei metodi di raccolta e di trattamento dei dati, agli indici impiegati per misurare la diseguaglianza, al numero di paesi inclusi nel campione, al periodo considerato. Alcune misure della diseguaglianza globale mostrano una sostanziale stabilità, altre un aumento, altre ancora una diminuzione. La diseguaglianza appare muoversi, nel tempo, lungo 
traiettorie non ben definite ed in modo irregolare. Nei movimenti di lungo periodo della diseguaglianza interna ai singoli paesi è tuttavia possibile identificare alcune regolarità in relazione all'intensificarsi dei processi di globalizzazione.

Le differenze nei livelli medi di reddito tra le aree ricche e quelle povere del pianeta sono oggi ancora molto elevate, e quindi concorrono in larga misura - unitamente alla diseguaglianza interna ai vari paesi - a determinare la diseguaglianza globale. La diminuzione della distanza fra Cina ed India da una parte e i paesi industrializzati dall'altra non è stata quindi sufficiente a compensare il mancato avvicinamento agli stessi da parte di altre aree dell'Asia, dell'America Latina e dell'Africa. Molto significativo è ancora il divario tra le distribuzioni dei redditi dei diversi paesi. E questo sia in termini di diseguaglianza globale sia con riferimento ai diversi gruppi di percettori.

La diseguaglianza misurata come disuguaglianza "internazionale" è diminuita in relazione alla crescita del reddito pro capite di paesi popolosi come la Cina e l'India. La componente between della diseguaglianza globale resta la componente principale dell'indice di diseguaglianza globale, sia che si calcoli l'indice di Gini o l'indice di Theil.

La localizzazione resta, dunque, un fattore molto importante nel determinare il livello dei redditi all'interno dei singoli paesi, e dunque anche la diseguaglianza esistente nella distribuzione degli stessi redditi All'inizio degli anni 2000 il peso relativo della componente tra paesi della diseguaglianza era nettamente superiore a quello della componente interna, evidenziando quindi un fenomeno significativamente diverso rispetto al XIX secolo, quando più del 40 per cento della diseguaglianza globale era spiegata da quella interna ai sistemi nazionali. Alcuni paesi poi, in passato classificati come in via di sviluppo (in particolare Cina e India), hanno sperimentato incrementi significativi nella dimensione della classe media segnalando la tendenza ad una progressiva riduzione della diseguaglianza interna. Altri invece, come molti paesi africani, continuano a presentare una significativa polarizzazione nella distribuzione dei redditi.

Le differenze nelle diverse distribuzioni dei redditi risultano ancora più accentuate a causa della diversità delle politiche attuate all'interno dei diversi sistemi per ridurre il livello di diseguaglianza. Esiste, dal punto di vista delle politiche redistributive, una forte asimmetria tra paesi industrializzati e paesi in via di sviluppo. Molti dei paesi che appartengono al primo gruppo, ed in particolare in quelli europei, 
intraprendono politiche per ridurre il grado di diseguaglianza. Tuttavia l'esperienza degli anni più recenti insegna che, in periodi di crisi, la possibilità di attuare politiche redistributive efficaci è limitata dai vincoli costituiti dalla necessità di ridurre i livelli del debito pubblico e di mantenere gli equilibri di bilancio. Nello stesso tempo la riduzione delle aliquote marginali sui redditi più elevati, adottata per rendere più uniforme la tassazione, per combattere l'evasione, nonché per impedire la fuga dei capitali verso i "paradisi fiscali", ha finito con l'accrescere la diseguaglianza nella distribuzione personale dei redditi (Brandolini et al., 2011). Nei paesi in via di sviluppo, d'altra parte, le politiche redistributive sono molto limitate e di scarsa efficacia. Il problema è tanto più grave, in quanto proprio in questi paesi il livello di povertà è ancora molto elevato.

$\mathrm{Ci}$ si deve interrogare allora se non sia possibile, e giustificabile dal punto di vista etico, individuare ed applicare delle politiche idonee a ridurre la diseguaglianza globale. Così come avviene all'interno delle singole nazioni, anche a livello internazionale, dovrebbero operare meccanismi redistributivi al fine di ridurre diseguaglianze di reddito così elevate. La domanda che immediatamente si pone è, dunque, quali politiche potrebbero essere implementate per ridurre divari che dipendono dalla localizzazione e dalla cittadinanza. Questi divari sono destinati a ridursi, almeno parzialmente, grazie alla crescita di paesi come la Cina e l'India, ma anche di altri paesi in via di sviluppo. Tuttavia in assenza di questo avvicinamento spontaneo sarebbe necessario individuare ed attuare politiche redistributive adeguate. Una riflessione sulla natura degli aiuti da parte delle Istituzioni internazionali e sulla direzione dei flussi potrebbe essere opportuna.

Una via, in un certo senso "spontanea" attraverso cui le differenze reddituali possono essere attenuate è certamente l'emigrazione. Le rimesse degli emigrati sono oggi una componente importante del reddito di alcuni paesi poveri. I flussi migratori, tuttavia, generano esternalità negative sia nei paesi di partenza che in quelli di arrivo. Si verifica, innanzituto, un vero e proprio impoverimento del capitale umano nei paesi esportatori. Emigra generalmente la quota di popolazione più preparata o quantomeno dotata di maggior spirito d'iniziativa. Nel paesi d'arrivo, poi, possono sorgere importanti problemi di integrazione non sempre facili da gestire. Una considerazione importante riguarda l'effettivo miglioramento nel tenore di vita degli immigrati che spesso è inferiore alle attese. E questo perché non è facile trovare una nuova 
occupazione o semplicemente perchè il potere d'acquisto dei redditi ottenuti nel paese d'arrivo è in realtà più basso di quanto possa essere stimato calcolando i redditi a parità dei poteri d'acquisto (PPP).

\section{BIBLIOGRAFIA}

Anand S. e P. Segal (2008), What do we know about global income inequality?, Journal of Economic Literature, 46(1), pp. 57-94.

Aron R. (1961), The Dawn of Universal History, Praeger Publisher, Westport, Connecticut, disponibile su http://books.google.it/books/about/The_Dawn_ of_Universal_History.html?id=RBxWAAAAYAAJ\&redir_esc $=y$

Arrow K.J. e G. Debreu (1954), Existence of an equilibrium for a competitive economy, Econometrica, 22 (3), pp. 265-90, July.

Atkinson A.B. (1997), Bringing Income Distribution in from the Cold, The Economic Journal, vol. 107 (441), pp. 297-321. March.

Atkinson A., T. Piketty e E. Saez (2011), Top Incomes in the Long Run of History, Journal of Economic Literature, 49(1), pp. 3-71.

Bhagwati J. (2004), In Defense of Globalization, Oxford University Press, New York (trad.it. Elogio della globalizzazione, Editori Laterza, Bari, 2004).

Bourguignon F. (2011), A turning point in global inequality..... and beyond, 2011, disponibile su http://siteresources.worldbank.org/EXTABCDE/Resources/74556761292528456380/7626791-1303141641402/7878676-1306270833789/ParallelSession-6-Francois_Bourguignon.pdf

Bourguignon F. (2012), The Globalization of Inequality, Moscow, April. http://www.slideshare.net/NewEconomicSchool/franois-bourguignonthe-globalization-ofinequality

Bourguignon F. (2013), Inclusive global growth: a framework to think about the post 2015 agenda, Angus Maddison Lecture, Oecd, Paris, April, disponibile su http://www.oecd.org/dev/pgd/Maddison-Oecd-April13_v2.pdf

Bourguignon F. and C. Morrisson (2002), The size distribution of income among world citizens, 1820-1990, American Economic Review, September, pp. 727-744.

Brandolini A., Micklewright J., Nolan B. e S.P. Jenkins (2011), The Great Recession and the Distribution of Household Income, FRDB, Palermo, settembre, http://www. frdb.org/upload/file/report_1_palermo.pdf

Cornia G.A. (2004), Changes in the distribution of income over the last two decades: extent, sources and possible causes, Rivista Italiana degli Economisti, n. 3, pp. 349-387.

Dollar D. e A. Kraay (2004), Trade, Growth, and Poverty, The Economic Journal, 114 (493), pp. F22-F49.

Glyn A. (2009), Functional Distribution and Inequality, in Salverda W., Nolan B e T. Smeeding T (editors), (2011), The Oxford Handbook of Economics Inequality, Oxford University Press, Oxford, pp. 71-100.

IMF (2008), Globalization: A Brief Overview, IMF Staff, may, disponibile su http://www.imf.org/external/np/exr/ib/2008/053008.htm

Kanbur R. e N. Lustig (1999), Why is Inequality Back on the Agenda?, Cornell 
University, Department of Agricultural Resource, and Managerial Economics WP 99-14, July, http://www.dyson.cornell.edu/research/researchpdf/wp/ 1999/Cornell_Dyson_wp9914.pdf

Krugman P.R. (2007), Does Outsourcing Change Everything?, Fifth Luca D'Agliano Lecture in Development Economics, June, disponibile su: http://www.dagliano.unimi.it/media/Lecture_5_text.pdf

Luxembourg Income Study (LIS, anni vari), http://www.lisdatacenter.org/

Luxembourg Wealth Study, LWS (anni vari), http://www.lisdatacenter.org/ourdata/lws-database/

Milanovic B. (2006a) Global Income Inequality: What It is and why It Matters, World Bank Policy Research Working Paper 3865, march, disponibile su http:// www.wds.worldbank.org/servlet/WDSContentServer/WDSP/IB/2006/03/02/0 00016406_20060302153355/Rendered/PDF/wps3865.pdf

Milanovic B. (2006b) Global Income Inequality: A Review, World Economics, 7 (1), pp. 131-157.

Milanovic B. (2009), Global inequality recalculated: The effect of new 2005 PPP estimates on global inequality, Policy Research Working Paper 5061, September, disponibile su http://elibrary.worldbank.org/doi/book/10.1596/1813-9450-5061? query $\mathrm{ID}=32 \% 2 \mathrm{~F} 6990040$

Milanovic B. (2011), Global Inequality. From Class to Location, from Proletarians to Migrants, World Bank Policy Research Working Paper 5820, September. http://www-wds.worldbank.org/servlet/WDSContentServer/WDSP/IB/2011/ 09/29/000158349_20110929082257/Rendered/PDF/WPS5820.pdf

Milanovic B. (2012), Global Income Inequality by the Numbers: in History and Now, An Overview, World Bank Policy Research Working Paper 6259, November, disponibile su http://www-wds.worldbank.org/servlet/WDSContentServer/WDSP/ IB/2012/11/06/000158349_20121106085546/Rendered/PDF/wps6259.pdf

Nagel T. (2005), The Problem of Global Justice, Philosopby and Public Affairs, 33 (2), pp. 113-147.

OECD (2007), OECD Workers in the Global Economy: Increasingly Vulnerable?, OECD Employment Outlook, July, disponibile su http://www.oecd.org/els/ employmentpoliciesanddata/38597305.pdf

OECD (2011a), An Overview of Growing Income Inequalities in OECD Countries: Main Findings, in: Divided We Stand, Why Inequality Keeps Rising, pp. 147http://www.oecd.org/els/soc/dividedwestandwhyinequalitykeepsrising.htm

OECD (2011b), Special Focus: Inequality in emerging economies (EEs), in: Divided We Stand, Why Inequality Keeps Rising, pp. 48-63, disponibile su http://www. oecd.org/els/soc/49170475.pdf

OECD (2013), Crisis squeezes income and puts pressure on inequality and poverty, New results from the OECD income distribution database, disponibile su http://www. oecd.org/social/soc/OECD2013-Inequality-and-Poverty-8p.pdf

Piketty T. e E. Saez (2007), Income and Wage Inequality in the USA, in Atkinson A.B. e T. Piketty (eds), Top Incomes over the Twentieth Century, Oxford University Press, Oxford, pp. 141-226.

Pogge T. (1994), An Egalitarian Law of Peoples, in "Philosophy and Public Affairs, 23 (3), pp. 195-224. 
Pogge T. (2008), World Poverty and Human Rights, $2^{\text {nd }}$. Edition, Cambridge Polity Press.

Pogge T. e S.J. Reddy (2010) How not to count the poor', in J. Stiglitz, P. Segal and S. Anand (Eds), Debates in the Measurement of Global Poverty, Oxford University Press, Oxford, pp. 42-85.

Rawls J. (1971), A Theory of Justice, Harvard University Press,Cambridge, Mass. (trad.it. Una teoria della giustizia, Feltrinelli, Milano, 1991).

Rawls J. (1999), The Law of Peoples, Harvard University Press, Cambridge, Massachusset, (trad.it. Il diritto dei popoli, Edizioni di Comunità, Torino, 2001).

Reddy S.J. (2005), The Role of Apparent Constraints in Normative, Reasoning: a Methodological Statement and Application to Global Justice, in "The Journal of Ethics" 9 (1), pp. 119-125

Reddy S.J. (2011), Economics and Human Rights: A Non-conversation, in "Journal of Human Development and Capabilities" 12, (1), pp. 63-72.

Robbins L. (1932), An Essay on the Nature and Significance of Economic Science, London: MacMillan.

Sala-i-Martin X. (2002), The Disturbing 'Rise' of Global Income Inequality, NBER Working paper No. 8904, april, disponibile su: www.nber.org.

Salverda W., B. Nolan e T.M. Smeeding (2009) (editors), The Oxford Handbook of Economic Inequality, Oxford Univeristy Press, Oxford.

Sen A.K. (1994), La diseguaglianza. Un riesame critico, $1^{\circ}$ ed., Il Mulino, Bologna (trad. it. di Inequality Reexamined, Oxford University Press, Oxford, 1992).

Sen A.K. (2001), Lo sviluppo è libertà. Perchè non c'è crescita senza democrazia, Arnoldo Mondatori Editore S.p.A., Milano (trad.it. di Development as freedom, New York: Alfred A. Knopf, 1999).

Sen A.K. (2002), Globalizzazione e libertà, Arnoldo Mondatori Editore S.p.A., Milano.

Sen A.K. (2010), L'idea di giustizia, Mondadori, Milano, (trad. it. di The Idea of Justice, Allen Lane, Penguin Books, 2009).

Singer P. (2004), One World: The Ebtics of Globalization, $2^{\text {nd }}$. Edition, New Haven, CT: Yale University Press.

Solow R.M. (1956), A contribution to the Theory of Economic Growth, The Quarterly Journal of Economics, 70 (1), pp. 65-94.

Somaini E. (2002), Uguaglianza. Teorie, politiche, problemi, Donzelli editore, Roma.

Stiglitz J. (2002), La globalizzazione e $i$ suoi oppositori, Giulio Einaudi editore, Torino (trad. it. di Globalization and Its Discontents, New York: W.W. Norton \& Company, 2002).

Targetti Lenti R. (2010), L'idea di giustizia per Amartya K. Sen, Nuova Antologia, Ottobre-Dicembre, pp. 320-332.

UNU-WIDER (2008), World Income Inequality Database, United Nations University's World Institute for Development Research, disponibile su: http://www.wider. unu.edu/wiid/wiid.htm.

Van Parijs P. (1995), Real freedom for all, Oxford University Press, Oxford.

Veca S. (2001), Sull'idea di giustizia globale, Scritti-Writings del Centro di filosofia sociale, Università di Pavia, disponibile su: http://cfs.unipv.it/opere/veca/etica.htm 
